\title{
Bilateral Optic Nerve Hypoplasia
}

National Cancer Institute

\section{Source}

National Cancer Institute. Bilateral Optic Nerve Hypoplasia. NCI Thesaurus. Code C101268.

A cong enital abnormality characterized by the underdevelopment of both optic nerves. 